\title{
Changes in Corneal Endothelial Cells Morphology Due to Different Cataract Surgery Procedures: A Retrospective Study From November 2014 to November 2016
}

\author{
MA BARIAH ${ }^{\mathrm{a}}$, LEONG SY \\ ${ }^{a}$ Optometry and Vision Sciences Program, Faculty of Health Sciences, Universiti Kebangsaan Malaysia, Jalan \\ Raja Muda Abdul Aziz, 50300 Kuala Lumpur, Malaysia. \\ ${ }^{b}$ Optometry and Vision Sciences Program, Faculty of Health Sciences, Universiti Kebangsaan Malaysia, Jalan \\ Raja Muda Abdul Aziz, 50300 Kuala Lumpur, Malaysia.
}

\section{ABSTRACT}

Introduction: The corneal endothelium is made up of an extremely thin layer of non-regenerating cells essential in keeping the cornea clear. The purpose of this study was to quantify the corneal endothelial cells morphology, visual acuity (VA) and refractive error in patients having femtosecond laser-assisted cataract surgery (FLACS) and conventional phacoemulsification surgery (CPS). Methods: Clinical data from 56 eyes were included in this study. 28 eyes had FLACS surgery and another 28 eyes had CPS. Distance VA, refractive error, corneal thickness and endothelial cells morphology were evaluated pre-operatively and at 1 month follow up. Refractive error was determined subjectively and VA was taken using Snellen chart. Specular microscope was used to measure endothelial cells morphology and central corneal thickness. Mann Whitney test and Spearman correlation analysis were used to analyse the results. Results: There were no statistically significant difference in the mean endothelial cell density $(p=0.24)$, mean central corneal thickness $(p=0.68)$, mean coefficient of variance $(p=0.19)$, mean hexagonality $(p=0.44)$, VA $(p=0.72)$ and postoperative refractive outcome $(p=0.66)$ between both groups. The duration of surgery was noted to have no statistically significant difference between both groups $(p=0.08)$, while the cumulative dissipated energy and torsional time showed a significant difference between groups $(p=0.01$ and $p<0.01$ respectively). Conclusion: This study concluded that there was no significant difference in the endothelial cells morphology in patients undergoing both cataract surgeries. Both surgeries presented similar outcomes in the patients' eyes.

KEYWORDS: Femtosecond laser-assisted cataract surgery (FLACS), conventional phacoemulsification surgery (CPS), endothelial cells density (ECD)

\section{INTRODUCTION}

The human cornea is made up of a transparent dome-like disc found on the anterior segment of the eye and it provides approximately two-thirds of the total refractive power of the eye. ${ }^{1}$ The corneal endothelium lies in the innermost layer of the

Corresponding author:

Bariah Mohd Ali

Optometry and Vision Sciences Program

Faculty of Health Sciences

Universiti Kebangsaan Malaysia

Jalan Raja Muda Abdul Aziz, 50300 Kuala Lumpur

Tel: 603-92897495 Fax: 603-26910488

Email: bariah@medic.ukm.my cornea. It is made up of a single layer of densely packed polygonal cells facing the anterior chamber and functions to maintain the corneal transparency. ${ }^{2,3}$ The normal endothelial cell count falls between 3000 to 3500 cells $/ \mathrm{mm}^{2}$ but it declines steadily to 2500 cells $/ \mathrm{mm}^{2}$ thereafter 80 years old. ${ }^{4-}$ ${ }^{6}$ However a minimum of 1000 cells $/ \mathrm{mm}^{2}{ }^{5,7}$ or at least 400 to 500 cells $/ \mathrm{mm}^{2}{ }^{6}$ of corneal endothelial cells density are required prior to cataract surgery in order to minimise the risk of corneal oedema, bullous keratopathy and loss of vision immediately after surgery or during the lifetime. ${ }^{5,7}$

The endothelial cells density measurement is important to recognise any potential of endothelial 
dysfunction or thinning before the surgery and it could also offer better information for the optometrist and the surgeon to identify the prognosis of the surgeries. ${ }^{8}$ The specular microscope is an instrument that provides a direct quantification of the corneal endothelial cells to assist in clinical decisions. ${ }^{9}$

Cataract is one of the most common age-related eye diseases which is seen among older patients ${ }^{10}$ and it equally affects both sexes. ${ }^{11}$ Based on the latest assessment done by the World Health Organisation (WHO) in year 2010, cataract remains the leading cause of blindness which is responsible for $51 \%$ or about 20 million people. ${ }^{12}$ This figure was supported by the survey done in Malaysia where there was 2.3 fold increment in the number of cataract surgeries done from year 2002 to $2011 .^{13}$

Hence, the development of advanced cataract surgery is not only simplifying the surgical procedures but it also helps towards achieving better visual outcome with optimal safety and minimum invasiveness. ${ }^{14}$ Previously, all the cataract surgeries were performed manually by the surgeons until the new introduction of femtosecond laser to assist cataract surgery. ${ }^{14}$

The Femtosecond Laser-assisted Cataract Surgery (FLACS) machine utilises pre-laser and real time optical coherence tomography images (OCT) and a single-piece curved $13 \mathrm{~mm}$ diameter disposable applanating lens and suction ring. ${ }^{15}$ These new features allow the surgeons to perform the three main surgical steps which are the capsulorhexis, lens fragmentation and liquefaction, corneal incision and arcuate corneal incision with the aid of femtosecond laser and the computer-controlled precision during the cataract surgery. ${ }^{15}$ After the laser procedure, a standard manual phacoemulsification and intraocular lens is inserted into the eye. ${ }^{15,16}$

Unlike FLACS, a blade cut incision is made during CPS and the lens nucleus is break up into tiny pieces by an ultrasonic probe through the small incision followed by the insertion of a tiny folderable $\mathrm{IOL}$ into the eye through that incision. ${ }^{17}$

Although cataract surgery is one of the safest and most successful major surgical procedures performed worldwide ${ }^{16}$ but it is well known that the application of ultrasound frequency during phacoemulsification can lead to corneal endothelial cells damage due to mechanical trauma caused from sonic waves and thermal injury. ${ }^{5,17-19}$ Therefore, the main advantages of FLACS is to decrease the effective phaco-time and reduction of phacoemulsification energy required in the cataract surgery. ${ }^{17}$

This study aims to investigate the changes in corneal endothelial cells morphology in patients undergoing Femtosecond Laser-assisted Cataract Surgery (FLACS) by using LenSx and conventional phacoemulsification surgery (CPS) in a private ophthalmology clinic. This machine is only available in private ophthalmology clinics in Malaysia and it provides an alternative surgical management for cataract patients.

\section{MATERIALS AND METHODS}

This is a retrospective study using data obtained from the clinical records of patients who attended Southern Specialist Eye Centre in Melaka from November 2014 to November 2016. This research followed the ethics approval obtained from the Universiti Kebangsaan Malaysia Research and Ethics Committee (UKM PPI/111/8/JEP-2016-660) and adhered to the Helsinki Declaration.

Initially, permission was obtained to review the medical records. Then, the patients were selected by using purposive sampling method where all the eligible patients were selected based on the inclusion and exclusion criteria as listed. The inclusion criteria included patients who underwent first time cataract surgery either using FLACS or CPS between November 2014 to November 2016 and patients with only posterior capsular intraocular lens implantation. To avoid bias, data from the surgical outcome of one surgeon were used in this study. Patients were treated with dispersive ophthalmic viscosurgery device (OVD), sodium hyaluronate $3.0 \%$ : chondroitin sulfate $4.0 \%$ (Viscoat, Alcon Laboratories (SA) Pty Ltd, Randberg, South Africa), incomplete data due to poor cooperation in diagnostic tests or non-compliance at follow up were excluded from this study.

Patients' demographic data such as age, gender and ethnicity were recorded in this study. Other clinical data including i) vision (distance VA was measured 
with Snellen chart, subjective refraction, auto kreading recorded using Accuref-K 9001 (SHINNIPPON Ryusyo Co.,Ltd. Japan) and cataract density was graded subjectively by the same experience surgeon based on LOC III system), ii) morphology of endothelial cells (endothelial cells density, central corneal thickness, coefficient of variance and hexagonality) was measured using a noncontact specular microscope (SP-1P, Topcon Medical Systems, Inc., Oakland, NJ), iii) axial length and anterior chamber depth was measured with IOLMAster V.5 (Carl Zeiss MeditecTnc. Germany), iv) percentage of endothelial cells loss was recorded and $v$ ) types of surgery were included. The efficacy parameters including cumulative dissipated energy (CDE), ultrasound torsional time (TT), total time of cataract procedure from the opening to closing of corneal incision as well as any complications were also recorded. All the measurements were performed by the optometrists who work in the Southern Specialist Eye Centre and data was collected and analysed by the authors.

The percentage of endothelial cells loss was calculated following the study by Conrad-Hengerer et al.(2013): ${ }^{2}$

$$
\text { Loss of } E C C(\%)=\frac{E C C \text { pre }-E C C \text { post }}{E C C \text { pre }} \times 100 \%
$$

\section{Statistical Analysis}

All the descriptive statistical analysis was performed using the IBM Corp. SPSS Statistics for Windows, Version 22 Armonk, NY: IBM Corp. The Wilcoxon $\mathrm{Z}$ test was used to compare the pre and post clinical data while Mann Whitney $U$ test was used to analyse the changes between both groups. Correlation analysis was performed using the Spearman's Rho correlation coefficient $\left(r_{s}\right)$. In all analyses, $p<0.05$ was considered statistically significant.

\section{RESULTS}

Data from 56 eyes were collected from 51 patients who fulfilled the inclusion criteria. Out of them, 34 were females and 22 were males. A total of 28 eyes were operated using FLACS and another 28 eyes were operated with CPS. The age range among the cataract surgeries patients was between 40 to 75 years in both groups. All the surgeries went well and none of the cataract surgery methods had caused complications during or after the cataract surgeries.

Table 1. shows the demographic data and baseline measurement of subjects in both groups. No significant difference was noted between measurements except for the axial length $(p=0.02)$ between both groups. Although the axial length in FLACS is significant longer than CPS group but the anterior chamber depth were almost similar between both groups.

Table 1. Patient demographics and baseline characteristics in the study

\begin{tabular}{llll}
\hline Parameter & FLACS & CPS & $p$-value \\
\hline $\begin{array}{l}\text { Age (years } \\
\text { old) }\end{array}$ & $63.25 \pm 8.24$ & $64.32 \pm 7.32$ & 0.72 \\
$\begin{array}{l}\text { Cataract } \\
\text { density }\end{array}$ & $2.03 \pm 0.74$ & $2.25 \pm 0.44$ & 0.15 \\
$\begin{array}{l}\text { Axial length } \\
\text { (mm) }\end{array}$ & $24.41 \pm 1.99$ & $23.23 \pm 1.02$ & $0.02 *$ \\
$\begin{array}{l}\text { Anterior } \\
\text { chamber } \\
\text { depth (mm) }\end{array}$ & $3.16 \pm 0.48$ & $2.98 \pm 0.42$ & 0.18 \\
\hline${ }^{*} p<0.05$ is considered significant &
\end{tabular}

${ }^{*} p<0.05$ is considered significant

Table 2. displays the results of endothelial cells density (ECD), endothelial cells loss (ECL), central corneal thickness (CCT), corneal coefficient of variance (CV) and corneal hexagonality (Hex) measurements. Preoperative mean ECD was lower in FLACS group $\left(2723.75\right.$ cells $\left./ \mathrm{mm}^{2} \pm 523.72\right)$ than in the CPS group ( 2863.89 cells $/ \mathrm{mm}^{2} \pm 372.83$ ), with no statistically significant difference $(p=0.46)$ between both groups. At one month follow up, the postoperative mean ECD measurements were higher in FLACS group $\left(2357.96\right.$ cells $\left./ \mathrm{mm}^{2} \pm 534.78\right)$ than CPS (2347.46 cells $/ \mathrm{mm}^{2} \pm$ 497.61) but it was not significantly difference between them $(p=0.24)$. Even though the mean percentage of ECL was higher in the CPS group $(17.77 \%)$ than in the FLACS group $(13.84 \%)$ but it was also no statistically significant $(p=0.44)$ observed.

Preoperative mean CCT values were similar between FLACS and CPS (521.11 um \pm 30.10 and 528.11 um \pm 35.33 respectively, $p=0.47$ ). However, at one month post-operative follow up, the FLACS showed CCT thickening (522.18 um \pm 37.24$)$ while CPS showed reduced in thickness (527.57 um \pm 56.59 ), but no statistical difference was detected between the two groups $(p=0.68)$. 
Table 2. Parameters measured at baseline and at 1 month post operation.

\begin{tabular}{|c|c|c|c|}
\hline Parameter & FLACS & CPS & $p$-value \\
\hline \multicolumn{4}{|l|}{ Endothelial cell count (cells $/ \mathrm{mm}^{2}$ ) } \\
\hline Baseline & $2723.75 \pm 523.72$ & $2863.89 \pm 372.83$ & 0.46 \\
\hline 1 month post-operative & $2357.96 \pm 534.78$ & $2347.46 \pm 497.61$ & 0.24 \\
\hline \multicolumn{4}{|l|}{ Endothelial cell loss (\%) } \\
\hline 1 month post-operative & $13.84 \pm 9.98$ & $17.77 \pm 14.71$ & 0.45 \\
\hline \multicolumn{4}{|l|}{ Central corneal thickness (um) } \\
\hline Baseline & $521.11 \pm 30.10$ & $528.11 \pm 35.33$ & 0.47 \\
\hline 1 month post-operative & $522.18 \pm 37.24$ & $527.57 \pm 56.59$ & 0.68 \\
\hline \multicolumn{4}{|l|}{ Corneal coefficient of variance (\%) } \\
\hline Baseline & $33.82 \pm 4.82$ & $35.50 \pm 5.15$ & 0.32 \\
\hline 1 month post-operative & $33.64 \pm 4.89$ & $32.61 \pm 5.61$ & 0.19 \\
\hline \multicolumn{4}{|l|}{ Corneal hexagonality (\%) } \\
\hline Baseline & $46.93 \pm 16.33$ & $46.36 \pm 16.32$ & 0.93 \\
\hline 1 month post-operative & $44.11 \pm 15.19$ & $28.43 \pm 20.23$ & 0.44 \\
\hline \multicolumn{4}{|l|}{ Visual Acuity } \\
\hline Baseline & $0.45 \pm 0.32$ & $0.41 \pm 0.26$ & 0.59 \\
\hline 1 month post-operative & $0.58 \pm 0.19$ & $0.55 \pm 0.22$ & 0.72 \\
\hline \multicolumn{4}{|l|}{ Refractive outcome (S.E) } \\
\hline 1 month post-operative & $-0.69 \pm 0.50$ & $-0.60 \pm 0.32$ & 0.66 \\
\hline Operation Duration (min) & $15.71 \pm 2.24$ & $15.00 \pm 0.00$ & 0.08 \\
\hline Cumulative dissipated Energy (U/s) & $7.63 \pm 6.00$ & $10.26 \pm 5.71$ & $0.01^{*}$ \\
\hline Torsional Time (Khz) & $0.90 \pm 1.97$ & $1.02 \pm 1.44$ & $<0.01^{*}$ \\
\hline
\end{tabular}

Preoperative mean CCT values were similar between FLACS and CPS (521.11 um \pm 30.10 and 528.11 um \pm 35.33 respectively, $p=0.47$ ). However, at one month post-operative follow up, the FLACS showed CCT thickening $(522.18 \mathrm{um} \pm 37.24)$ while CPS showed reduced in thickness (527.57 um \pm 56.59$)$, but no statistical difference was detected between the two groups $(p=0.68)$.

Preoperative mean CV values were almost similar between FLACS and CPS $(33.82 \% \pm 4.82$ and $35.50 \% \pm$ 5.15 respectively, $p=0.32)$. At one month postoperative follow up, FLACS $(33.64 \% \pm 4.89)$ and CPS $(32.61 \% \pm 5.61)$ showed no significant difference in mean CV changes $(p=0.19)$ between both groups. There was also no statistically significant difference in the preoperative Hex between FLACS and CPS $(46.93 \% \pm 16.33$ and $46.36 \pm 16.31$, respectively, $p=0.93)$. At one month post-operative follow up, the mean Hex has reduced to $44.11 \% \pm 15.19$ by FLACS and $28.43 \% \pm 20.23$ by CPS but no significant corneal hexagonality changes were noted between both groups $(p=0.44)$.

Baseline distance VA was similar between FLACS and CPS $(0.45 \pm 0.32$ and $0.41 \pm 0.26$ respectively, $p=0.59)$. Although the mean VA showed improvement at one month post-operative follow up in FLACS group $(0.58 \pm 0.19)$ and CPS group $(0.55 \pm$ 0.22 ) but there were no significant differences between the two groups $(p=0.72)$. Despite of that, the mean spherical equivalence at one month postoperative was higher in the FLACS group $(-0.69 \mathrm{D} \pm$ $0.50)$ than the CPS group $(-0.60 D \pm 0.32)$ but no statistically significant difference was showed between groups $(p=0.66)$.

The mean operation duration used for FLACS was 15.71 mins \pm 2.24 and 15.00 mins \pm 0.00 by using CPS has showed no significant difference between both groups $(p=0.08)$, unlike the cumulative dissipated energy and torsional time showed a 
significant difference between both groups $(p=0.01$ and $p<0.01$ respectively).

\section{DISCUSSION and CONCLUSION}

The study investigated retrospectively the impact of two different cataract surgeries (FLACS and CPS) on the corneal endothelial cells morphology, VA and refractive error carried out by one surgeon. The results showed insignificant difference between the two types of surgeries in all the measurements conducted. Despite the axial length in FLACS being significantly longer than CPS group, the anterior chamber depth was almost similar between both groups. The reason to secure an adequate anterior chamber depth is important for preserving the corneal endothelial cells loss from the mechanical and thermal damage during the phacoemulsification procedure.

The post-operative uncorrected VA and refractive outcomes obtained from the FLACS and CPS in this study showed no significant difference between both groups. These findings were similar to Roberts et al. (2013) and Chen et al. (2014). ${ }^{16,20}$ Chen et al. (2014) reviewed the VA and refractive outcomes of FLACS. ${ }^{20}$ They summarised that the majority of the authors found the VA and refractive outcomes of FLACS to be statistically equivalent to those of CPS.

Roberts et al. (2013) also found no significant difference in refractive outcomes in a prospective study of 113 FLACS procedures versus 105 CPS cases. ${ }^{16}$ The absolute mean difference was $0.29 \mathrm{D} \pm$ 0.25 for FLACS group and $0.31 \mathrm{D} \pm 0.24$ for the CPS group ( $p=0.51)$ and more than $90 \%$ of patients in both groups achieved 6/12 uncorrected distance VA three months postoperatively.

Hence, the findings of VA and refractive outcomes between FLACS and CPS in this study were possible due to the absence of pre-operative complications such as A-scan measurement errors or intraoperative complications like anterior capsular tear and IOL tilts. According to Abdelghany \& Alio (2014) the most common complication encountered following cataract surgery is residual refractive error resulting in suboptimal visual outcomes and which was possible due to preoperative, operative or postoperative causes. ${ }^{21}$

During phacoemulsification surgery, the generated heat and fluid turbulence within the anterior chamber causes damage to the endothelial cells. This is probably the reason why there was a reduction of ECD noted in our study similar to the Takács et al. (2012) and Gros-Otero et al. (2013) studies. ${ }^{22,23}$ Furthermore, no dispersive ophthalmic viscosurgery device (Viscoat) was used to protect the endothelial cells during the cataract procedures in this study which caused further loss of ECD.

Although the remaining post-operative mean ECD in both groups were less than that found in Takács et al. (2012) and Gros-Otero et al. (2013) $)^{22-23}$ studies but it was still above the minimum baseline suggested by Wilson \& Poper-Hall (1982), Ventura et al. (2001) and Soliman et al. (2012). ${ }^{5-7}$ The analysis of this study showed no significant difference in ECD changes post-operatively between both groups but we observed higher percentage of endothelial loss and change in cell hexagonality 1 month postoperatively in CPS than FLACS group of subjects. It is possible that lower ultrasound energy used in FLACS results in lesser corneal endothelial cells damage.

Since Takács et al. (2012), Gros-Otero et al. (2013) and Krarup et al. (2014) studies showed higher ECD were preserved postoperatively in patients treated with FLACS than CPS but all these studies presented with insignificant difference of corneal endothelial cell loss between both groups. ${ }^{22-24}$ Takács et al. (2012) found an insignificant reduction of 123 cells/ $\mathrm{mm}^{2}(4.4 \%)$ by FLACS and 299 cells $/ \mathrm{mm}^{2}(10.5 \%)$ by CPS, Gros-Otero et al. (2013) found $-4.70 \% \pm 15.78 \%$ loss of endothelial cell density in CPS group and $4.24 \% \pm 15.04 \%$ in FLACS group and Krarup et al. (2014) reported the mean of endothelial cell loss at 3 months was 274 cells $/ \mathrm{mm}^{2} \pm 358(11.4 \%)$ by using FLACS and 333 cells $/ \mathrm{mm}^{2} \pm 422$ (13.9\%) by using CPS method. ${ }^{22-24}$

Based on the findings in the study conducted by Takács et al. (2012), this could be due to the author did not include dense cataract (grade 4+) or white cataract which may require more phacoemulsification energy to break the lens during the surgery, Gros-Otero et al. (2013) concluded that his findings obtained could be possible due to smaller cohort and the used of dispersive ophthalmic viscosurgery device during surgery. ${ }^{22,23}$ Krarup et al. (2014) also reported an insignificant mean of endothelial cell loss at 3 months could be because the author classified the eye with most dense 
cataract was operated with FLACS and the eye with less cataract with CPS. ${ }^{24}$

There was also no significant difference in CCT between both groups. According to Yu et al. (2015) and Takács et al (2012), a significant difference in CCT between both groups were only seen on the first day post-operatively, but the difference became insignificant one month into the surgery in either group. ${ }^{19,22}$ Since our data was obtained one month post-operatively, the outcome of this study agreed with the findings of the earlier mentioned studies.

Regarding association, there was no significant relationship shown between VA and percentage of endothelial cells loss in both groups. This observation was probably because none of the techniques caused any severe endothelial cells loss resulting in corneal oedema or pseudophakic bullous keratopathy that can adversely affect postoperative VA. Roberts et al. (2013) and Krarup et al. (2014) showed no significant VA changes and endothelial cells loss in their patients but no relationship was found between the two variables in their studies. ${ }^{16,24}$

In conclusion, this study showed that there was no significant difference in the endothelial cells morphology, refractive status and VA postoperatively between cataract patients who were operated either using FLACS or CPS techniques. However more data is needed to support these findings.

\section{ACKNOWLEDGEMENT}

Sincere thanks to Dr. Robert Yeo Kim Chuan, medical director of Southern Specialist Eye Centre in Melaka and his medical team members for the efforts and supports to provide the completed cataract surgeries patients' medical records.

\section{REFERENCES}

1. Zavala J, Jaime GL, Barrientos CR, ValdezGarcia J. Corneal endothelium: developmental strategies for regeneration. Eye 2013; 27:57988.

2. Conrad-Hengerer I, Al Juburi M, Schultz $T$, Hengerer FH, Dick HB. Corneal endothelial cell loss and corneal thickness in conventional compared with femtosecond laser-assisted cataract surgery: three-month follow-up. Journal of Cataract \& Refractive Surgery 2013; 39:1307-13.

3. Navaratnam J, Utheim TP, Rajasekhar VK, Shahdadfar A. Substrates for expansion of corneal endothelial cells towards bioengineering of human corneal endothelium. Journal of functional biomaterials 2015; 6:917-45.

4. Smolin G, Thoft RA. The cornea: scientific foundations and clinical practice: Little, Brown; 1983.

5. Soliman MA, Eid MZ, Abdel-Badei M, Hafez A, Bhatia J. Relationship between endothelial cell loss and microcoaxial phacoemulsification parameters in noncomplicated cataract surgery. Clinical Ophthalmology 2012; 6:503-10.

6. Ventura AS, Wälti R, Böhnke M. Corneal thickness and endothelial density before and after cataract surgery. British journal of ophthalmology 2001; 85:18-20.

7. Wilson R, Roper-Hall M. Effect of age on the endothelial cell count in the normal eye. The British journal of ophthalmology 1982; 66:513.

8. Bonnell AJ, Cymbor M. Under specular the microscope: take a closer look at the clinical and financial benefits that non-contact specular microscopy could offer your practice. Review of Optometry 2012; 149:58-64.

9. Brightbill FS, MCDonnell PJ, McGhee CN, Farjo AA, Serdarevic O. Corneal Surgery: Theory Technique and Tissue: Elsevier Health Sciences; 2008.

10. Ontario HQ. Intraocular lenses for the treatment of age-related cataracts: an evidence -based analysis. Ontario health technology assessment series 2009; 9:1.

11. Onal S, Bavbek T. Aging and the eye. Marmara Med J. 2005; 18:43-52.

12. Organization WH. Prevention of blindness and visual impairment. Priority eye diseases-corneal opacities http://www.who.int/blindness/ causes/priority/en/index9.html (accessed Jul 2014). 2010.

13. The Fifth Report of The National Eye Database. Association of Clinical Registries, Malaysia 2001.

14. Lee M-Y, Goh P-P, Salowi MA, Adnan TH, Ismail $M$. The Malaysian Cataract Surgery Registry: Cataract Surgery Practice Pattern. The AsiaPacific Journal of Ophthalmology 2014; 3:343-7.

15. Abouzeid, H. \& Ferrini, W. 2014. Femtosecond-Laser Assisted Cataract Surgery: A 
Review. Acta Ophthalmologica 92(7):597-603.

16. Roberts TV, Lawless $M$, Chan CC, Jacobs M, Ng

$D$, Bali SJ, et al. Femtosecond laser cataract surgery: technology and clinical practice.

Clinical \& experimental ophthalmology 2013; 41:180-6.

17. Chen X, Xiao W, Ye S, Chen W, Liu Y. Efficacy and safety of femtosecond laser-assisted cataract surgery versus conventional phacoemulsification for cataract: a metaanalysis of randomized controlled trials. Scientific reports 2015; 5.

18. Lundberg $B$, Jonsson $M$, Behndig $A$. Postoperative corneal swelling correlates strongly to corneal endothelial cell loss after phacoemulsification cataract surgery. American journal of ophthalmology 2005; 139:1035-41.

19. Yu A, Ni LY, Wang QM, Huang F, Zhu SQ, Zheng $L Y$, et al. Preliminary clinical investigation of cataract surgery with a noncontact femtosecond laser system. Lasers in surgery and medicine 2015; 47:698-703.

20. Chen, H., Hyatt, T. \& Afshari, N. 2014. Visual and Refractive Outcomes of Laser Cataract Surgery. Current Opinion in Ophthalmology 25 (1): 49.

21. Abdelghany AA, Alio JL. Surgical options for correction of refractive error following cataract surgery. Eye and Vision 2014; $1: 2$.

22. Takács Ál, Kovács I, Miháltz K, Filkorn T, Knorz MC, Nagy ZZ. Central corneal volume and endothelial cell count following femtosecond laser-assisted refractive cataract surgery compared to conventional phacoemulsification. Journal of refractive surgery 2012; 28:387-91.

23. Gros-Otero, J., Contreras-Martin, I., BlazqueSanchez, V., Hurtado-Cena, F.J., Garcia-Perez, J., Merino-Hijosa, M.J., and Alvarez-

Rementeria, L., Induced Changes In Macula and Endothelium After Femtosecond Laser-assisted Cataract Surgery: Comparison With Standard Phacoemulsification. J Emmetropia 2013; 4:185 $-189$.

24. Krarup T, Morten Holm L, la Cour M, Kjaerbo H. Endothelial cell loss and refractive predictability in femtosecond laser-assisted cataract surgery compared with conventional cataract surgery. Acta ophthalmologica 2014; 92:617-22. 
